\title{
Conductas de riesgo, síntomas depresivos, auto y heteroagresión en una muestra de adolescentes escolarizados en la Región Metropolitana de Santiago de Chile, 2007
}

Risk behaviors, depressive symptoms, auto and heteroaggresion in a sample of adolescent students in Metropolitan Santiago de Chile, 2007

Ramón Florenzano U. ${ }^{1,2,4,5}$, Eugenio Cáceres C. ${ }^{1 a}$, Macarena Valdés C. ${ }^{2 b}$, Silvia Calderón S. ${ }^{2 c}$, Sylvia Santander R. ${ }^{3}$ y Martín Casassus T. ${ }^{4 d}$.

The aim of this study is to corroborate a reference framework that relates parental styles with drugs consumption, depression, self and other aggressive behaviors. We found that parents' acceptance correlates positively with adolescents' initiative and negatively with depressive and suicidal symptoms; psychological control with depression and behavioral control with antisocial behavior. Material and Methods: The CNAP+ (Cross National Adolescent Program) questionnaire (version CNAP Plus) was adapted in interaction with its author; and then was applied to a representative, random, three-stages sample of the Metropolitan Region $(n=1.447)$. Results: The worse the relation with parents, the higher the consumption, depression, suicidal ideation and hetero aggressive behaviors. Spearman's correlation coefficients were found in the same direction of a previous transcultural CNAP study. Frequent consumption of alcohol was found in $26.9 \%$, of tobacco in $31.2 \%$, of marihuana in $6.9 \%$ and of cocaine or other drugs in $2.3 \%$ of the sample studied. The same sample has suicide ideation $7.76 \%$ and depressive symptoms $6.9 \%$. Self aggression prevailed among women and hetero aggression among men. With age there was an increase in alcohol consumption $\left(\chi^{2}=107.95, p=0.0001\right)$, of tobacco $\left(\chi^{2}=63.02, p=0.0001\right)$, of marihuana $\left(\chi^{2}=18.78, p=0.001\right)$ and of cocaine $\left(\chi^{2}=8.995\right.$, $p=0.06)$. There is differential by age in violent behaviors, suicide ideation or depressive

Recibido: 2 de enero de 2009

Aceptado: 15 de marzo de 2009

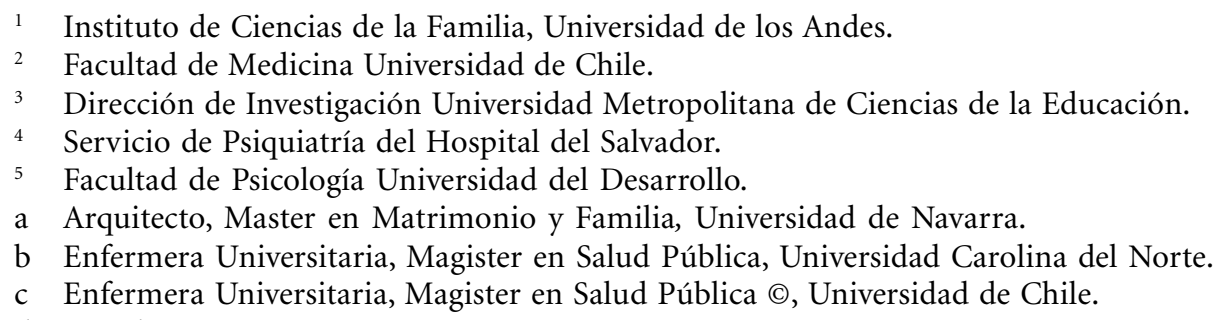

Trabajo realizado con un subsidio del Fondo de Apoyo a la Investigación (FAI) de la Universidad de los Andes. ( proyecto FAI-MED 003-007) 
symptoms. Conclusion: The results obtained by Barber et al in the multicultural application of his model in 16 countries is confirmed in this study run in Santiago de Chile. This is relevant to consider when designing public policies and preventive interventions. This survey confirms previous studies regarding sex, age and parental styles; there is higher consumption of chemical substances and hetero aggressive conducts among men, and of depressive feelings and suicide ideation among women. The studied behaviors increase with age, except for violence; they also correlate with each other and decrease when there is a good relation with parents.

Key words: Adolescence, Risk conducts, parental styles.

Rev Chil Neuro-Psiquiat 2009; 47 (1): 24-33

\section{Introducción}

Las conductas de riesgo adolescentes son objeto de preocupación creciente, y diversos estudios chilenos e internacionales han documentado su aumento en los últimos años ${ }^{2,3}$. En publicaciones previas hemos analizado los cambios en la frecuencia de estos comportamientos juveniles, en las últimas décadas, así como la influencia de la familia en su aparición ${ }^{4}$, como un foco en el diseño de programas preventivos. Entre los modelos utilizados para estudiar la interacción familiaadolescente vale la pena mencionar el de protección-riesgo desarrollado por Jessor y Jessor en la década de los 70 y luego complementado con los factores escalada-resiliencia por Blum y otros autores. En los 90 se adaptó en Chile y la Organización Panamericana de la Salud difundió en América, los instrumentos ECRA, CTF y CSF (Escala de Conductas de Riesgo Adolescentes, Cómo es Tu Familia y Cómo es Su Familia) para cuantificar esta interacción. En la Universidad de Harvard, Stuart Hauser agregó los conceptos de familias habilitadoras y restrictivas y mostró como pueden aumentar o disminuir la frecuencia de estas conductas. En los últimos 10 años el Prof. Brian Barber de la Universidad de Tennessee ha planteado un modelo interaccional, que se ha validado en un estudio transcultural, a través de la batería de escalas CNAP (Cross National Adolescent Program). Barber describe diversas dimensiones de estilos de parentalidad y sus efectos en los hijos adolescentes. Según este autor la parentalidad tiene dos componentes: uno de apo- yo y aceptación y otro de control definido por una gama de conductas reguladoras y disciplinarias. Estas dimensiones de la parentalidad se basan en las descripciones originales de Schaefer ${ }^{5,6}$ 1965b) quien identificó tres dimensiones denominadas "aceptación vs rechazo", "control vs autonomía xpsicológica" y "control firme vs control laxo". La distinción entre control psicológico y conductual reflejaría una organización que afecta significativamente el funcionamiento del joven. La dimensión de "Aceptación-Rechazo" se caracteriza en su polo positivo por compartir, expresar afecto, evaluación positiva, etc y en el polo negativo por descuido, negligencia y rechazo; la dimensión "Control Psicológico y Salud Mental" se refiere al control parental intrusivo que interfiere en el desarrollo psicológico y emocional del niño (e. g., invalidación de sentimientos, restricción de expresiones verbales, retiro de amor e inducción de culpabilidad entre otras); y finalmente, la de "control firme $v s$ control laxo" se define por "dejar hacer" con autonomía extrema en un polo y castigo y estrictez en el polo contrario. Schaefer (1965b) explica que esta dimensión indica el grado en que los padres establecen reglas y regulaciones, ponen límites a las actividades del niño y hacen cumplir tales reglas y regulaciones.

De acuerdo a lo descrito, las asociaciones de variables posibles son:

(a) las conductas de apoyo parental estarían relacionadas positivamente con la competencia interpersonal del adolescente, y el grado en el cual los adolescentes inician interacciones y relaciones con sus pares y adultos; 
(b) el control psicológico intrusivo de los padres tendría una asociación positiva con niveles más bajos de salud mental de los adolescentes, y específicamente síntomas depresivos y

(c) el control conductual de los padres estaría negativamente relacionado a la inconformidad, medida por conductas antisociales de los adolescentes lo que puede explicarse de dos maneras distintas: una de ellas señala que el control parental facilita la autorregulación necesaria para que los niños inhiban conductas trasgresoras y se involucren en conductas socialmente aprobadas en tanto la otra indica que los niños que carecen de supervisión tienen más probabilidad de ser influenciados por sus pares, algunos de los cuales podrían fomentar conductas riesgosas y desviadas.

Este modelo ha sido empíricamente corroborado con la aplicación del instrumento CNAP por el equipo de la Universidad de Tennesee ${ }^{7}$ en un estudio transcultural en 16 países distintos, y los autores en conjunto con el Prof Barber han realizado su adaptación chilena.

Este estudio ha planteado la existencia en la cultura chilena de asociaciones entre la conducta apoyadora parental y la manifestación de iniciativa social en los adolescentes, entre el control psicológico parental y síntomas depresivos en los adolescentes y entre el bajo control de conductual de los padres y las manifestaciones de conductas antisociales en sus hijos adolescentes (Me parece que esta hipótesis, la que anima esta investigación según entiendo, debiera quedar formulada en el resumen del paper).

En esta publicación describimos la aplicación de la encuesta CNAP en una versión adaptada para Chile (CNAP Plus) enfocada, en especial, a la presencia de depresión y de conductas auto y heteroagresivas y al estudio de las diferencias entre adolescentes menores de hasta 15 años y adolescentes mayores entre los 16 y 18 años, así como el consumo diferencial de drogas por edad y género. Finalmente, evaluaremos si es posible replicar las asociaciones del modelo de Barber a través de correlaciones biseriadas de Spearman entre las diversas variables que lo componen.

\section{Material y Métodos}

Tal como se ha señalado, se adapta una nueva versión en castellano, del instrumento C NAP, desarrollado en un estudio transcultural por Barber et al entre 1999 y 2004, en cuatro reuniones de trabajo conjuntas con el autor (Bellaggio, Italia - 2006; Santiago de Chile y Concepción 2007). El instrumento adaptado (CNAP Plus) es piloteado en cuatro países: Chile, Bolivia, México y España. Se presentan los resultados de la primera aplicación de este instrumento, a una muestra aleatoria trietápica (por comuna, establecimiento y curso) de estudiantes pertenecientes a 14 establecimientos de enseñanza media de la Región Metropolitana, seleccionados a partir del listado de establecimientos educacionales del MINEDUC, sorteando comunas estratificadas por nivel socio-económico, luego establecimientos municipales, particulares subvencionados y privados, y finalmente cursos dentro de esos establecimientos. En la Tabla 1 se lista la cantidad de encuestas aplicadas en los catorce establecimientos, para llegar al número de 1.447 adolescentes estudiados. La encuesta fue aplicada por estudiantes de Pedagogía de la Universidad Metropolitana de Ciencias de la Educación, previamente capacitados por uno de los co-autores.

\section{Resultados}

Los 1.447 estudiantes encuestados tenían una edad promedio de 15,8 años (DS 1,66) y un $58,2 \%$ eran mujeres. Presentaban consumo frecuente de alcohol (varios días a la semana) un 26,9\%, de tabaco un $31,2 \%$, de marihuana un $6,9 \%$ y de cocaína u otras drogas duras un $2,3 \%$. El consumo de alcohol y drogas duras es mayor entre los varones y no hay diferencias de género en consumo de tabaco o marihuana (Tablas 2 y 3 ). Ha presentado ideación suicida un 7,3\% y síntomas depresivos un 6,5\% (Tablas 4 y 5), con una frecuencia mayor entre las mujeres y ascendente hasta los 18 años para la depresión y hasta los 16 para la suicidalidad. En cuanto a conductas 
Tabla 1. Distribución de frecuencias y porcentajes de estudiantes encuestados en Región Metropolitana, Santiago 2007

\begin{tabular}{ccr}
\hline Establecimiento & $\begin{array}{c}\text { n de estudiantes } \\
\text { encuestados }\end{array}$ & $\%$ \\
\hline 1. A & 61 & 4,2 \\
2. B & 107 & 7,4 \\
3. C & 44 & 3,0 \\
4. D & 156 & 10,8 \\
5. E & 88 & 6,1 \\
6. F & 53 & 3,7 \\
7. G & 86 & 5,9 \\
8. H & 142 & 9,8 \\
9. I & 76 & 5,3 \\
10. J & 122 & 8,4 \\
11. K & 162 & 11,2 \\
12. L & 126 & 8,7 \\
13. M & 155 & 10,7 \\
14. N & 69 & 4,7 \\
Total & 1.447 & 100,0 \\
\hline
\end{tabular}

hetero-agresivas, éstas aumentan con la edad (Tabla 6) y son significativamente más frecuentes en el sexo masculino (Tabla 7). Presentan riñas individuales frecuentes un $5,4 \%$, grupales un 3,9\%, conductas vandálicas un $5,9 \%$ y robos menores un $2,5 \%$.

Al comparar por género se encontraron diferencias significativas en el consumo de sustancias químicas, con mayor uso frecuente

* de alcohol entre los hombres $(33,3 \%$ vs $22,2 \%$ entre las mujeres) $\left(\chi^{2}=25,53, \mathrm{p}=0,0001\right)$,

* de marihuana $(17,3 \%$ de los hombres vs $9,9 \%$ de las mujeres) $\left(\chi^{2}=32,888, p=0,0001\right) y$

* de cocaína u otras drogas $(5,3 \%$ de los hombres $v s \quad 1,3 \%$ de las mujeres) $\left(\chi^{2}=21,439, \mathrm{p}=\right.$ $0,0001)$. En cambio, no hubo diferencias por género en el uso de tabaco y sí las hubo en las conductas hetero-agresivas:

* robos menores $(12,2 \%$ de los hombre vs $5,5 \%$ de las mujeres) $\left(\chi^{2}=31,749, \mathrm{p}=0,0001\right)$;

Tabla 2. Consumo semanal o más comparado por género, 1.447 adolescentes RM, 2007, expresado en porcentajes

\begin{tabular}{lcccc}
\hline C Semanal+ & $\begin{array}{c}\text { Hombres } \\
(\mathbf{n = 1 9 5})\end{array}$ & $\begin{array}{c}\text { Mujeres } \\
(\mathbf{n = 1 8 3})\end{array}$ & $\chi^{\mathbf{2}}$ & $\mathbf{p}$ \\
\hline Alcohol & $33,30 \%$ & $22,20 \%$ & 21,255 & 0,0001 \\
Tabaco & $30,80 \%$ & $30,50 \%$ & 2,868 & 0,238 \\
Marihuana & $10,20 \%$ & $3,90 \%$ & 25,661 & 0,0001 \\
Drogas duras & $4,10 \%$ & $0,90 \%$ & 19,028 & 0,0001 \\
\hline
\end{tabular}

$\chi^{2}: 21,255,2 \mathrm{~g}, \mathrm{p}=0,0001$

Tabla 3. Consumo semanal o más comparado por edad en años, 1.447 adolescentes RM, 2007, expresado en porcentajes

\begin{tabular}{lcccc}
\hline Años de edad & Alcohol & Tabaco & Marihuana & Drogas duras \\
\hline Doce & 0 & 0 & 0 & 0 \\
Trece & 0 & 0 & 0 & 0 \\
Catorce & 11,6 & 14,3 & 2,2 & 0 \\
Quince & 17,6 & 23,8 & 4,3 & 1,1 \\
Dieciseis & 29,6 & 34,3 & 4,8 & 2,1 \\
Diecisiete & 38,6 & 41,3 & 8,5 & 2,4 \\
Dieciocho & 43,8 & 45,3 & 13,7 & 2,1 \\
Diecinueve & 50 & 50 & 6,2 & 0 \\
Veinte & 0 & 0 & 0 & 0 \\
& $\chi^{2}=140,652$ & $\chi^{2}=88,339$ & $\chi^{2}=79,384$ & $\chi^{2}=23,158$ \\
& $\mathrm{p}=0,0001$ & $\mathrm{p}=0,0001$ & $\mathrm{p}=0,0001$ & $\mathrm{p}=0,171$ \\
\hline
\end{tabular}


* riñas en el colegio $(20,2 \%$ de los hombres $v s$ $10,1 \%$ de las mujeres) $\left(\chi^{2}=39,037, \mathrm{p}=0,0001\right) \mathrm{y}$

${ }^{\star}$ peleas en pandillas $(16,1 \%$ de los hombres $v s$ $4 \%$ de las mujeres) $\left(\chi^{2}=62,476, p=0,0001\right)$.

Por otra parte, informan haber presentado

Tabla 4. Depresión e ideación suicida según edad, 1.447 adolescentes RM Santiago de Chile, 2007

\begin{tabular}{lcc}
\hline Edad & $\begin{array}{c}\text { Frecuente } \\
\text { depresión } \\
\%\end{array}$ & $\begin{array}{c}\text { Ideación } \\
\text { suicida } \\
\%\end{array}$ \\
\hline Doce & 0 & 0 \\
Trece & 4,80 & 4,80 \\
Catorce & 6,60 & 4,50 \\
Quince & 7,30 & 7,50 \\
Dieciseis & 4,80 & 24,50 \\
Diecisiete & 7,60 & 10,80 \\
Dieciocho & 7,40 & 5,10 \\
Diecinueve & 7,10 & 1 \\
Total & 6,50 & 7,30 \\
& $\chi^{2}=9,127$ & $\chi^{2}=17,183$ \\
& $\mathrm{p}=0,908$ & $\mathrm{p}=0,511$ \\
\hline
\end{tabular}

tristeza o depresión de moderadas a severas, un $30,6 \%$ de los hombres vs un 39\% de las mujeres $\left(\chi^{2}=63,02, \mathrm{p}=0,004\right)$ y han pensado frecuentemente en matarse un $6 \%$ de los hombres vs un $8,9 \%$ de las mujeres $\left(\chi^{2}=9,725, \mathrm{p}=0,008\right)$.

Al comparar el consumo frecuente de sustancias químicas y la percepción de los jóvenes de su relación con la madre y el padre (Tabla 8), se encuentra en forma estadísticamente significativa que cuando esta es percibida negativamente,

Tabla 5. Depresión e ideación suicida según sexo, 1.447 adolescentes RM Santiago de Chile, 2007

\begin{tabular}{lcc}
\hline Género & $\begin{array}{c}\text { Depresión } \\
\%\end{array}$ & $\begin{array}{c}\text { Ideación } \\
\text { suicida } \\
\%\end{array}$ \\
\hline Hombres & 7 & 6 \\
Mujeres & 7,30 & 8,90 \\
Total & 7,00 & 7,70 \\
& $\chi^{2}=11,008$ & $\chi^{2}=9,725$ \\
$\mathrm{p}=0,004$ & $\mathrm{p}=0,008$ \\
\hline
\end{tabular}

Tabla 6. Conductas heteroagresivas según edad, adolescentes RM Santiago de Chile 2007

\begin{tabular}{lcccccccccc}
\hline & $\mathbf{1 2}$ & $\mathbf{1 3}$ & $\mathbf{1 4}$ & $\mathbf{1 5}$ & $\mathbf{1 6}$ & $\mathbf{1 7}$ & $\mathbf{1 8}$ & $\mathbf{1 9 +}$ & $\chi^{\mathbf{2}}$ & $\mathbf{p}$ \\
\hline Vandalismo & 0 & 0 & 4,4 & 5,9 & 6,1 & 6,5 & 4,2 & 7,1 & 10,635 & 0,909 \\
Robo mayor & 0 & 0 & 1,6 & 1,6 & 2,9 & 1 & 3,2 & 7,1 & 14,549 & 0,693 \\
Robo menor & 0 & 0 & 2,2 & 2,4 & 1,6 & 1,4 & 3,1 & 7,1 & 11,555 & 0,869 \\
Fuga del hogar & 0 & 4,8 & 5,5 & 3,3 & 2,6 & 3,1 & 4,2 & 4,2 & 18,804 & 0,404 \\
Peleas grupales & 0 & 4,8 & 3,3 & 4,6 & 6,3 & 4,8 & 3,1 & 7,1 & 11,573 & 0,869 \\
Peleas individuales & 0 & 4,3 & 6 & 5,9 & 4,2 & 5,1 & 6,3 & 7,1 & 9,764 & 0,939 \\
\hline
\end{tabular}

Tabla 7. Conductas heteroagresivas según edad, adolescentes RM Santiago de Chile 2007

\begin{tabular}{llclc} 
& \multicolumn{2}{c}{ Semanal o más } & & \\
& $\begin{array}{c}\text { Varón } \\
\%\end{array}$ & $\begin{array}{c}\text { Mujer } \\
\%\end{array}$ & $\chi^{2}$ & p \\
\hline Vandalismo & 9,70 & 3,10 & 68,801 & 0,0001 \\
Robo mayor & 5,10 & 0,50 & 45,438 & 0,0001 \\
Robo menor & 4,40 & 0,70 & 30,028 & 0,0001 \\
Fuga del hogar & 6,30 & 2,20 & 17,37 & 0,0001 \\
Peleas grupales & 8 & 3,40 & 34,444 & 0,0001 \\
Peleas individuales & 9,80 & 2,90 & 63,266 & 0,0001 \\
\hline
\end{tabular}


Tabla 8. Percepción de la relación con la madre, con el padre y consumo de sustancias químicas, expresada en porcentajes, 1.447 adolescentes RM Santiago de Chile, 2007

\begin{tabular}{|c|c|c|c|c|}
\hline \multirow[b]{2}{*}{ Consumo Semanal+ } & \multicolumn{2}{|c|}{ Relación con la madre } & \multirow[b]{2}{*}{$\chi^{2}$} & \multirow[t]{2}{*}{$\mathbf{p}$} \\
\hline & $\begin{array}{c}\text { Peor } \\
\%\end{array}$ & $\begin{array}{c}\text { Mejor } \\
\%\end{array}$ & & \\
\hline Alcohol & 11,00 & 24,80 & 8,6 & 0,01 \\
\hline Tabaco & 36,70 & 28,60 & 9,657 & 0,008 \\
\hline Marihuana & 8,20 & 6,00 & 13,654 & 0,001 \\
\hline Drogas duras & 3,50 & 1,60 & 12,139 & 0,002 \\
\hline \multirow[b]{2}{*}{ Consumo Semanal+ } & \multicolumn{2}{|c|}{ Relación con el padre } & & $\mathbf{p}$ \\
\hline & $\begin{array}{c}\text { Peor } \\
\%\end{array}$ & $\begin{array}{c}\text { Mejor } \\
\%\end{array}$ & $\chi^{2}$ & \\
\hline Alcohol & 30,10 & 23,50 & 7,54 & 0,02 \\
\hline Tabaco & 34,30 & 27,50 & 10,345 & 0,006 \\
\hline Marihuana & 9,20 & 4,60 & 20,67 & 0,0001 \\
\hline Drogas duras & 2,30 & 2,10 & 5,94 & 0,05 \\
\hline
\end{tabular}

se tiene un mayor consumo de todas las sustancias, con excepción del caso de consumo de alcohol y relación con la madre. La misma relación se encuentra para depresión y suicidalidad en la cual los puntajes aumentan a peor percepción de la relación (Tabla 9) y también para la presencia de conductas hetero-agresivas (Tabla 10).

Tabla 9. Depresión e ideación suicida y percepción de la relación con la madre, 1.447 adolescentes $R M$ Santiago de Chile, 2007

\begin{tabular}{lcc}
\hline $\begin{array}{l}\text { Relación con } \\
\text { la madre }\end{array}$ & $\begin{array}{c}\text { Depresión } \\
\%\end{array}$ & $\begin{array}{c}\text { Ideación } \\
\text { suicida } \\
\%\end{array}$ \\
\hline Peor & 13 & 15 \\
Mejor & 4,70 & 4,70 \\
Total & 7,00 & 7,70 \\
& $\chi^{2}=70,824$ & $\chi^{2}=63,424$ \\
& $\mathrm{p}=0,0001$ & $\mathrm{p}=0,0001$ \\
\hline Relación con & Depresión & $\begin{array}{c}\text { Ideación } \\
\text { suicida }\end{array}$ \\
el padre & $\%$ & $\%$ \\
\hline Peor & 11 & 13 \\
Mejor & 3,71 & 3,20 \\
Total & 7,00 & 7,70 \\
& $\chi^{2}=96,727$ & $\chi^{2}=80,971$ \\
& $\mathrm{p}=0,0001$ & $\mathrm{p}=0,0001$ \\
\hline
\end{tabular}

Al calcular una matriz de correlación de Spearman para las variables anteriores (Tabla 11), se encuentran coeficientes de correlación de Spearman positivos sobre +02 para el consumo de alcohol y tabaco $(0,557)$ y para la depresión e ideación suicida $(0,508)$

Otros datos significativos para similar correlación son los de:

- consumo de tabaco y de marihuana $(+0,372)$;

- consumo de alcohol y de marihuana $(+0,358)$;

- edad y consumo de alcohol (+0,309);

- vandalismo y sexo masculino $(0,220)$;

- tabaco y mayor edad $(0,207)$;

- consumo de marihuana y de drogas duras $(+0,388)$;

- consumo de marihuana con robo mayor $(+0,378)$, robo menor $(+0,269)$ y fuga del hogar $(+0,225)$

- consumo de drogas duras con vandalismo $(+0,210)$, robo mayor $(+0,494)$ y robo menor $(+0,225)$.

Cabe destacar, además, que la separación parental no alcanza correlaciones especialmente elevadas y que se identificaron coeficientes de correlación negativos para ideación suicida y calidad de relación con el padre $(-0,240)$ y con la madre $(-0,200)$ y de depresión con los mismos factores $(-0,223$ y $-0,228)$. 
Tabla 10. Conductas heteroagresivas según relación con la madre, 1.447 adolescentes RM Santiago de Chile, 2007

\begin{tabular}{|c|c|c|c|c|}
\hline \multirow[t]{2}{*}{ Relación con la madre } & \multicolumn{2}{|c|}{ Calidad relación } & \multirow[b]{2}{*}{$\chi^{2}$} & \multirow[b]{2}{*}{$\mathbf{p}$} \\
\hline & Peor & Mejor & & \\
\hline Vandalismo & $9,50 \%$ & $4,30 \%$ & 21,943 & 0,0001 \\
\hline Robo mayor & $4,80 \%$ & $1,60 \%$ & 25,428 & 0,0001 \\
\hline Robo menor & $3,80 \%$ & $1,80 \%$ & 7,959 & 0,01 \\
\hline Fuga del hogar & $7,50 \%$ & $2,50 \%$ & 22,818 & 0,0001 \\
\hline Peleas grupales & $8 \%$ & $4,20 \%$ & 10,62 & 0,005 \\
\hline Peleas individuales & $9,50 \%$ & $4,50 \%$ & 14,824 & 0,001 \\
\hline \multirow[t]{2}{*}{ Relación con el padre } & \multicolumn{2}{|c|}{ Calidad relación } & & \\
\hline & Peor & Mejor & $\chi^{2}$ & p \\
\hline Vandalismo & $6,50 \%$ & $5,80 \%$ & 2,31 & 0,315 \\
\hline Robo mayor & $3,20 \%$ & $2,10 \%$ & 5,353 & 0,069 \\
\hline Robo menor & $2,40 \%$ & $2,50 \%$ & 0,047 & 0,977 \\
\hline Fuga del hogar & $5,50 \%$ & $3,90 \%$ & 18,167 & 0,0001 \\
\hline Peleas grupales & $7 \%$ & $4,80 \%$ & 3,775 & 0,151 \\
\hline Peleas individuales & $7,00 \%$ & $5,30 \%$ & 1,642 & 0,441 \\
\hline
\end{tabular}

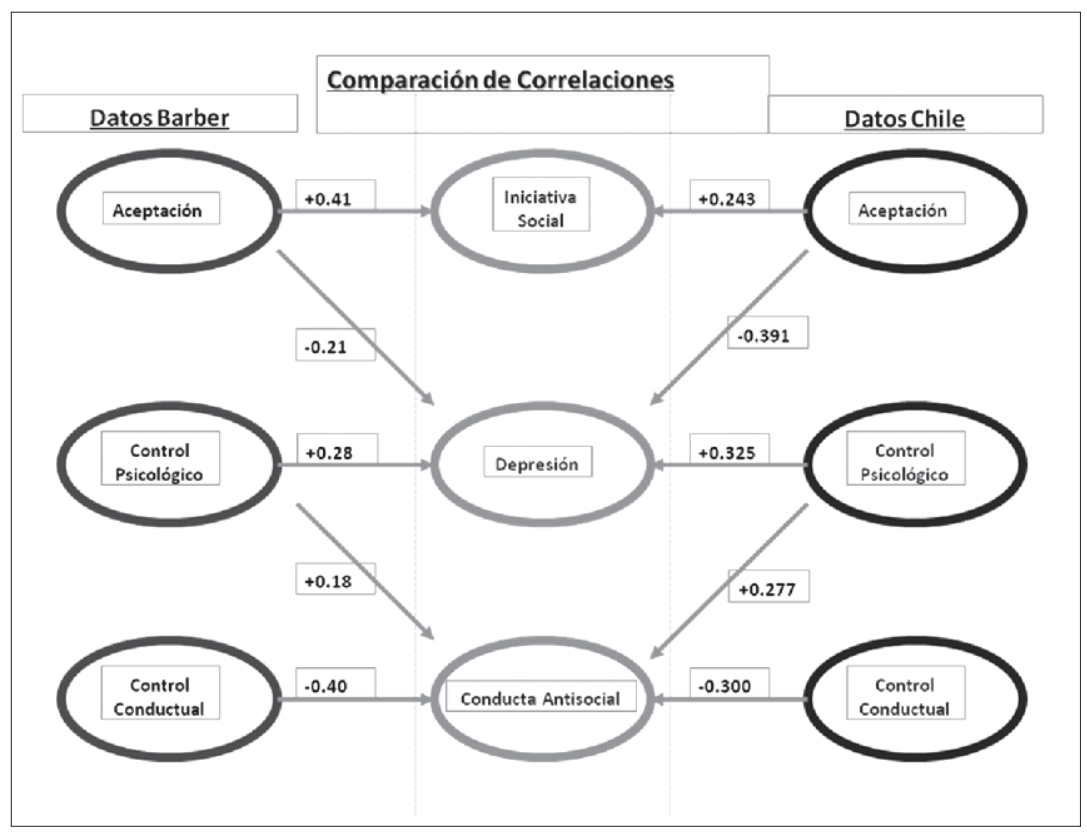

Figura 1. Comparación de correlaciones de Spearman en estudio CNAP de Barber (2004) y en encuesta CNAP Plus en Santiago de Chile, 2007.
La Figura 1 compara los coeficientes de correlación encontrados en este estudio (Tabla 11) con los realizados por el grupo de Barber (2004), mostrando como las correlaciones se dan en ambos casos, en la misma dirección.

\section{Conclusiones}

Una de las limitaciones de este estudio es su diseño de corte horizontal: al no seguir una cohorte prospectivamente, no se puede predicar 
Tabla 11. Coeficientes de correlación de Spearman, consumo de alcohol, conductas auto y heteroagresivas y variables familiares, 1.447 adolescentes RM Santiago de Chile, 2007

\begin{tabular}{|c|c|c|c|c|c|c|}
\hline & Depresión & Antisocial & Aceptación & Iniciativa & Control Psi & $\begin{array}{c}\text { Control } \\
\text { conductual }\end{array}$ \\
\hline \multicolumn{7}{|l|}{ Depresión } \\
\hline Correlación de Pearson & 1 &, $330(* *)$ &,$- 407(* *)$ &,$- 198(* *)$ &, $350\left({ }^{* *}\right)$ &,$- 255(* *)$ \\
\hline Sig. (bilateral) & & 0,000 & 0,000 & 0,000 & 0,000 & 0,000 \\
\hline $\mathrm{N}$ & 1.405 & 1.362 & 1.126 & 1.276 & 1.137 & 1.250 \\
\hline \multicolumn{7}{|l|}{ Antisocial } \\
\hline Correlación de Pearson &, $\left.330{ }^{* *}\right)$ & 1 &,$- 249\left({ }^{* *}\right)$ &,$- 075(* *)$ &, $281(* *)$ &,$- 309(* *)$ \\
\hline Sig. (bilateral) & 0,000 & & 0,000 & 0,007 & 0,000 & 0,000 \\
\hline $\mathrm{N}$ & 1.362 & 1.384 & 1.116 & 1.262 & 1.127 & 1.232 \\
\hline \multicolumn{7}{|l|}{ Aceptación } \\
\hline Correlación de Pearson &,$- 407(* *)$ &,$\left.- 249{ }^{* *}\right)$ & 1 &, $273\left({ }^{* *}\right)$ &,$- 428(* *)$ &, $522(* *)$ \\
\hline Sig. (bilateral) & 0,000 & 0,000 & & 0,000 & 0,000 & 0,000 \\
\hline $\mathrm{N}$ & 1.126 & 1.116 & 1.153 & 1.060 & 1.072 & 1.118 \\
\hline \multicolumn{7}{|l|}{ Iniciativa } \\
\hline Correlación de Pearson &,$- 198\left(^{* *}\right)$ &,$- 075\left(^{\star *}\right)$ &, $273\left({ }^{* *}\right)$ & 1 & $-0,046$ &, $230(* *)$ \\
\hline Sig. (bilateral) & 0,000 & 0,007 & 0,000 & & 0,130 & 0,000 \\
\hline $\mathrm{N}$ & 1.276 & 1.262 & 1.060 & 1.302 & 1.065 & 1.162 \\
\hline \multicolumn{7}{|l|}{ ControlPsi } \\
\hline Correlación de Pearson &, $\left.3500^{* *}\right)$ &, $281\left({ }^{* *}\right)$ &,$- 428\left(^{* *}\right)$ & $-0,046$ & 1 &,$- 279(* *)$ \\
\hline Sig. (bilateral) & 0,000 & 0,000 & 0,000 & 0,130 & & 0,000 \\
\hline $\mathrm{N}$ & 1.137 & 1.127 & 1.072 & 1.065 & 1.166 & 1.127 \\
\hline \multicolumn{7}{|l|}{ Control Conductual } \\
\hline Correlación de Pearson &,$- 255(* \star)$ &,$- 309(* \star)$ &, $522(* *)$ &, $230(* *)$ &,$- 279(* *)$ & 1 \\
\hline Sig. (bilateral) & 0,000 & 0,000 & 0,000 & 0,000 & 0,000 & \\
\hline $\mathrm{N}$ & 1.250 & 1.232 & 1.118 & 1.162 & 1.127 & 1.282 \\
\hline
\end{tabular}

causalidad. Si bien sería lógico pensar que los estilos de crianza parentales predisponen a la aparición de vivencias o conductas anormales, no es imposible que los adolescentes con psicopatología distorsionen su recuerdo de las interacciones de crianza con sus padres. Sólo un seguimiento longitudinal con una muestra mayor puede aclarar la direccionalidad de la asociación encontrada. Una segunda limitación son los moderados valores de las correlaciones encontradas, a pesar de ser estadísticamente significativas: ninguna supera $+0,5$.
Interesa comparar los resultados de este estudio con los datos del VII Estudio Nacional de Drogas de $\mathrm{CONACE}^{8}$ y si bien las categorías de consumo frecuente no son exactamente iguales entre ambos, se tienen cantidades muy cercanas en consumo frecuente de tabaco, alrededor de $31 \%$ en la aplicación del CNAP Plus y $35 \%$ en las estadísticas de CONACE referido al consumo de 20 días o más en el último mes.

En cuanto a diferencias por sexo, en este estudio el consumo de tabaco fue muy cercano entre ambos sexos (30,8\% en hombres y $30,5 \%$ en mu- 
jeres) en tanto en el de CONACE hubo mayor consumo femenino $(37,9 \%)$ que masculino $(32,8 \%)$ y el uso frecuente (semanal o más) de alcohol diferenció significativamente entre el $33,3 \%$ de los varones y $22,2 \%$ de las mujeres en la aplicación del CNAP Plus, mientras que CONACE encuentra cifras muy cercanas para ambos sexos para prevalencia de último mes (37,5\% entre hombres y $38,2 \%$ para mujeres).

En cuanto a uso frecuente de marihuana, este análisis identifica para una frecuencia semanal o más, de $10,2 \%$ para hombres y $3,9 \%$ para mujeres, mientras que el consumo frecuente según CONACE (10 o más veces en el año) es de 35,8\% para los hombres y $22,8 \%$ para las mujeres).

El aumento del consumo en la medida que se avanza en edad se puede comparar sólo indirectamente con CONACE que presenta los resultados por curso (desde $8^{\circ}$ básico a $4^{\circ}$ medio), pero las tendencias son semejantes: el consumo frecuente de alcohol según CNAP Plus aumenta desde $11,6 \%$ a los catorce años, a $50 \%$ a los diecinueve años, en tanto en el estudio de CONACE el aumento es de $18,4 \%$ en $8^{\circ}$ Básico a $50,4 \%$ en $4^{\circ}$ Medio y en el caso del tabaco, el aumento es de $14,3 \%$ a los catorce años, a $50 \%$ a los 19 y de $20,4 \%$ en $8^{\circ}$ Básico a $45,4 \%$ en $4^{\circ}$ Medio, respectivamente.

Para el uso frecuente de marihuana, el incre- mento en la aplicación del CNAP Plus es desde $2,2 \%$ a los 14 años a $13,7 \%$ a los 18 y la de CONACE se sube (consumo 10 o más veces al año), desde $29,3 \%$ en $8^{\circ}$ Básico a $32,7 \%$ en $4^{\circ}$ Medio y para el uso frecuente de cocaína el aumento es de $1,1 \%$ a los 14 años a $2,2 \%$ a los 18 y de $2,4 \%$ en $8^{\circ}$ Básico a $5,6 \%$ en $4^{\circ}$ Medio, respectivamente, corroborando la mayor frecuencia de consumo de sustancias químicas y conductas heteroagresivas entre los hombres y de sentimientos depresivos e ideación suicida entre las mujeres. Asimismo, se constata el aumento de las antedichas conductas en los adolescentes mayores. Se encuentran correlaciones de las diversas conductas de riesgo entre sí, así como su disminución cuando la relación percibida, especialmente con el padre y luego con la madre es buena. En un análisis posterior, se analizará el rol de los diversos estilos parentales en los indicadores de salud mental y conductuales de los adolescentes.

Este estudio ilustra la importancia de estudiar detalladamente los mecanismos proximales y distales de la interacción parento-filial, y sus hallazgos pueden ser importante para las políticas públicas tanto de juventud como de familia: tanto la falta de comunicación afectiva como el exceso de control psicológicos pueden tener efectos negativos. El monitoreo conductual parece ser útil para disminuir comportamientos arriesgados.

\section{Resumen}

Este estudio busca corroborar en Santiago de Chile el marco referencial de Brian Barber ${ }^{l}$ que relaciona consumo de drogas, depresión, suicidalidad y conductas violentas con estilos parentales. Encontramos que la aceptación de los padres se correlaciona positivamente con iniciativa adolescente y negativamente con sintomas depresivos y suicidas; el control psicológico con depresión, y el control conductual con conducta antisocial. Material y Métodos: En conjunto con su autor, se adapta el instrumento CNAP, Cross National Adolescent Program (Versión CNAP Plus) y se aplica a una muestra representativa aleatoria trietápica de la Región Metropolitana $(n=1.447)$. Resultados: A peor relación con los padres mayor consumo, depresión, ideación suicida, y conductas heteroagresivas. Se encuentran coeficientes de correlación de Spearman en la misma dirección de los del estudio internacional CNAP. Se encontró consumo frecuente de alcohol en 26,9\%, de tabaco en 31,2\%, de marihuana en 6,9\%, de cocaina u otras drogas en 2,3\% de la muestra estudiada. Hay en la misma muestra ideación suicida en 7,7\% y 
sintomas depresivos en 6,9\%. La autoagresión predomina en mujeres y la heteroagresión en varones. Con la edad aumenta el consumo de alcohol $\left(\chi^{2}=107,95, p=0,0001\right)$, de tabaco $\left(\chi^{2}=63,02, p=0,0001\right)$, de marihuana $\left(\chi^{2}=18,78, p=0,001\right)$ y de cocaina $\left(\chi^{2}=8,995\right.$, $p=0,06)$. No hay diferencias por edad en conductas violentas, ideación suicida o sintomas depresivos. Conclusiones: Los resultados obtenidos por Barber en la aplicación multicultural de su modelo en 16 países se confirman en este estudio en Santiago de Chile, lo que importa como base de información posible de considerar en el diseño de políticas públicas (estatales y privadas) $y$ de intervenciones preventivas. Esta encuesta confirma estudios previos sobre sexo, edad y estilos parentales: hay mayor consumo de sustancias químicas y conductas heteroagresivas entre los varones y de sentimientos depresivos e ideación suicida entre las mujeres. Las conductas estudiadas aumentan con la edad, con excepción de la violencia; estas conductas se correlacionan entre sí, y disminuyen con una buena relación con los padres.

Palabras clave: Adolescencia, conductas de riesgo, estilos parentales.

\section{Referencias}

1. Barber B K. Intrusive parenting: How psychological control affects children and adolescents. Washington, DC: American Psychological Association Press. 2002a.

2. CONACE. Séptimo Estudio Nacional de Drogas en Población General de Chile. Ministerio del Interior, Chile, 2006.

3. Santander S, Zubarew T, Santelices L, Argollo A, Cerda J, Borquez M. Influencia de la familia como factor protector de conductas de riesgo en escolares chilenos. Rev Méd Chile 2008; 136: 317-24.

4. Florenzano R, Cáceres E, Valdés M, Santander S, Calderón $\mathrm{S}$, Cassasus $\mathrm{M}$, et al. Conductas de riesgo y factores de protección en una muestra representativa de adolescentes escolarizados en la Re- gión Metropolitana. Actas del III Congreso Chileno de Adolescencia, Santiago de Chile 4 al 6 de Septiembre de 2008.

5. Schaefer E S. Children's reports of parental behavior: An inventory. Child Development 1965a; 36: 413-24.

6. Schaefer E S. A configurational analysis of children's reports of parent behavior. Journal of Consulting Psychology 1965b; 29: 552-7.

7. Barber B K, Stolz H E, Olsen J A. Parental support, Psychological control and Behavioral control: Assessing relevance across time, methods and culture, Monographs of the Society for Research in Child Development 2005; 70: 4.

8. Ministerio del Interior, CONACE. VII Estudio Nacional de Drogas en Población Escolar de Chile, 2007. $8^{\circ}$ Básico a $4^{\circ}$ Medio Informe de Principales Resultados. Santiago de Chile, 2008.

Correspondencia:

Ramon Florenzano Urzúa

Av. Plaza 700, 2037158

E-mail: rflorenzano@gmail.com 